\title{
MEASURING FIELDING PERFORMANCE IN CRICKET
}

\author{
PARAG SHAH \\ Gujarat University, H L College of Commerce, Department of Statistics \\ Mailing address: Parag Shah, H L College of Commerce, Department of Statistics, Navrangpura, Ahmedabad, \\ 380009, Gujarat, India, tel.: +91 98 25026247, fax: +91 79 26460595, e-mail: pbs1374@yahoo.co.in
}

\begin{abstract}
Introduction. In cricket, the evaluation of individual player performance has been based on measures such as batting and bowling averages. These statistics are used to quantify the batting and bowling performance of cricketers, but there are no statistics for measuring the performance of fielders. This paper introduces a measure that can be used to assess the fielding performance of cricketers. Method. Various factors that are considered important in fielding are quantified to scores based on the ball-by-ball information of a match for each cricketer. The fielding points of each ball are then combined to calculate the total fielding points of a cricketer in a given match. All the fielding points are then added in order to obtain total fielding points of a cricketer up to a given match. Average fielding points are obtained by dividing the total fielding score by the number of matches played. Data. To demonstrate these measures, the first ODI match of India against Zimbabwe played on $11^{\text {th }}$ June, 2016, is examined. Conclusion. The recommended measures can be used to quantify the fielding performances of cricketers for a series of matches, whether it is ODI or Twenty20 cricket. They make it possible to assess the average fielding performance of each player. Individual fielding performance scores can then be aggregated to measure the overall fielding performance of a team.
\end{abstract}

Key words: cricket, fielding score, average fielding score

\section{Introduction}

The skills of sportspersons are evaluated through performance statistics which indicate the level of achievement of the players [1]. Cricket has a number of performance-related statistics that are usually used to measure the performance of players, such as the strike rate and batting average for batsmen, and the bowling average, economy rate, and bowling strike rate for bowlers. A batting index was developed by Croucher [2] as the product of the batting average and strike rate, for rating players in one-day cricket. Lemmer [3] proposed a measure for assessing the performance of bowlers based on the three traditional performance statistics. To measure the performance of batsmen, Barr and Kantor [4] proposed a measure based on the strike rate and batting average, and another one was defined by Lemmer [5]. While selecting a limited overs cricket squad, Gerber and Sharp [6] defined various indices for batting, bowling, fielding, all-rounder performance, and wicket keeping separately. Saikia et al. [7], on the other hand, proposed a model that can be used to quantify the batting, bowling, or wicket-keeping performances of cricketers based on the data from the scorecard of a match. A fielder always tries to limit the number of runs that the batsman can score and also tries to get the batsman out by catching the ball or by executing a run-out.

Accomplishments in batting and bowling are usually mentioned in newspaper headlines, but good fielding can also make a crucial contribution to a team's success. With the advent of one-day internationals (ODI) and the recent format of Twen- ty20 cricket, fielding has become more athletic and competitive. Unlike in Test cricket, saving runs is almost as important as scoring runs in ODI and Twenty20 cricket. Fielders have to dive, make sliding stops, and throw in hard. If players perform well in the field, they can help their team to win the match. Two aspects of fielding are always noticeable, that is the number of catches taken and run-outs, but there are other aspects that are associated with these two factors. For example, a catch taken by a fielder may be simple or difficult. The first action is relatively easier to perform compared to the second one. In the case of a run-out, a fielder has to throw hard into the appropriate end, or, depending on the situation, hit the stumps directly. One has to collect the ball-by-ball information of a match to examine all these aspects of fielding. A measure that does not take all these important aspects into account is not suitable for determining the fielding performance of a cricketer. Therefore, in this paper, a performance statistic was present for quantifying the fielding performance of cricketers through the ball-by-ball information of a match.

\section{Method}

Three different aspects of fielding (i.e. catching, run saving, and running out) can be observed as far as the on-field performance of cricketers is concerned. A swift and active fielder always tries to catch the ball in one action and/or throw it as hard and quickly as possible, irrespective of the fielding position. An ex- 
ceptional catch or throw in a cricket match may even turn its results. If all these aspects of fielding are to be considered, the ball-by-ball information of a match is required, which can be obtained by observing the match live. In the approach presented in the current article, various parameters are considered, and points are assigned to these parameters based on the on-field performances of the fielders. The parameters and their corresponding points are given in table 1 .

Table 1. Points for each fielding parameter

\begin{tabular}{|c|c|c|c|c|c|c|}
\hline & Catch & $\begin{array}{c}\text { Run- } \\
\text { out }\end{array}$ & Stumping & $\begin{array}{c}\text { Catch } \\
\text { dropped }\end{array}$ & $\begin{array}{c}\text { Missed } \\
\text { run-out }\end{array}$ & $\begin{array}{c}\text { Missed } \\
\text { stumping }\end{array}$ \\
\hline Easy & 10 & 10 & 10 & -5 & -5 & -5 \\
\hline Difficult & 15 & 15 & 15 & 0 & 0 & 0 \\
\hline $\begin{array}{c}\text { Very } \\
\text { difficult }\end{array}$ & 20 & 20 & 20 & 0 & 0 & 0 \\
\hline
\end{tabular}

Actual runs saved because of fielding are added to the fielding points of the player, and actual runs missed due to misfielding are subtracted from the fielding points of the player.

Adding the fielding points gives the Total fielding points (TFP) of each player.

$$
T F P=\sum F P
$$

The Average fielding points (AFP) of a player equal TFP divided by the number of matches played.

$$
A F P=\frac{T F P}{\text { Number of matches played }}
$$

\section{Data}

Let us take the first ODI match of India against Zimbabwe played on $11^{\text {th }}$ June, 2016, to demonstrate how fielding points are calculated and tabulated in table 2 .

Table 2. Points obtained by each player in the ODI match

\begin{tabular}{|l|c|c|c|c|}
\hline \multicolumn{1}{|c|}{ Player } & Catch points & Run points & $\begin{array}{c}\text { Run-out/ } \\
\text { Stumping } \\
\text { points }\end{array}$ & $\begin{array}{c}\text { Fielding } \\
\text { points (FP) }\end{array}$ \\
\hline L. Rahul & $-5+10=5$ & 1 & 0 & 6 \\
\hline M. Pandey & 0 & $1-2=-1$ & 0 & -1 \\
\hline K. Nair & 0 & 0 & 0 & 0 \\
\hline A. Rayudu & 0 & $2-3=-1$ & 0 & -1 \\
\hline K. Jadhav & 0 & 0 & 0 & 0 \\
\hline M. Dhoni & 20 & $4+1=5$ & 0 & 25 \\
\hline A. Patel & 0 & 0 & 0 & 0 \\
\hline D. Kulkarni & 0 & 0 & 0 & 0 \\
\hline J. Bumrah & 0 & 0 & 0 & 0 \\
\hline B. Shran & -5 & -1 & 0 & -6 \\
\hline Y. Chahal & 0 & 0 & 0 & 0 \\
\hline
\end{tabular}

\section{Conclusion}

In the initial years of cricket, a player attained their place in the team either as a specialist batsman, a bowler, or an all-rounder. But in limited overs matches, fielding has gained significant importance, as the saving of runs, good catches, and runs-outs are key for winning matches. The captain of a fielding team tries to put their best fielders in the crucial positions in the field, so that they are likely to make difficult catches and be instrumental in bringing about a swift run-out that can significantly impact the result of the match. Therefore, to recognise a skilled fielder, it is essential to have a fielding performance measure based on the ball-by-ball information of the match. Even though collecting such information is tedious work, it is essential in order to quantify a player's fielding ability. The recommended measures can also be used to quantify the fielding performances of cricketers for a series of matches, whether it is ODI or Twenty 20 cricket. The average fielding performance of each player can be calculated. Also, individual fielding performance scores can be aggregated to assess the overall fielding performance of a team. An all-round measure of a player can be then obtained as an aggregate of their batting average, bowling average, and fielding average. Such a measure would be comprehensive, as it would include all the parameters of the game, namely those related to batting, bowling, and fielding.

\section{Literature}

1. Clarke S.R. (2007). Studying variability in statistics via performance measures in sport. Bulletin of the International Statistical Institute $56^{\text {th }}$ session, Lisbon.

2. Croucher J.S. (2000). Players' ratings in one-day cricket. Proceedings of the $5^{\text {th }}$ Australian Conference on Mathematics and Computers in Sport, 14-16 June 2000 (pp. 95-106). Sydney: Sydney University of Technology.

3. Lemmer H.H. (2002). The combined bowling rate as a measure of bowling performance in cricket. South African Journal of Research in Sports, Physical Education and Recreation 24(2), 37-44.

4. Barr G.D., Kantor B.S. (2004). A criterion for comparing and selecting batsmen in limited overs cricket. The Journal of the Operational Research Society 55(12), 1266-1274.

5. Lemmer H.H. (2004). A measure for the batting performance of cricket players. South African Journal for Research in Sport, Physical Education and Recreation 26(1), 55-64.

6. Gerber H., Sharp G.D. (2006). Selecting a limited overs cricket squad using an integer programming model. South African Journal for Research in Sport, Physical Education and Recreation 28(2), 81-90.

7. Saikia H., Bhattacharjee D., Bhattacharjee A. (2011). Is IPL responsible for cricketers' performance in Twenty20 World Cup? International Journal of Sports Science and Engineering 6(2), 96-110.

Submitted: June 11, 2016

Accepted: June 29, 2016 